Original Article (short paper)

\title{
The three and six-substitution rules in football: A preliminary comparative analysis in quantitative replacing, game statistics, win rate and winning probability
}

\author{
Carlos Fernando Braz Ribeiro ${ }^{1}$ (D), Leonardo dos Santos Siqueira $^{2}$ (D), Dalton Pinheiro Pinto ${ }^{3,4}$ (D), \\ Cristiano Diniz da Silva ${ }^{2}$ \\ ${ }^{1}$ Universidade Federal de Viçosa, Centro de Ciências Biológicas, Departamento de Educação \\ Física, Curso de Especialização de Futebol, Viçosa, MG, Brasil. \\ ${ }^{2}$ Universidade Federal de Juiz de Fora, Instituto de Ciências da Vida, Departamento de \\ Educação Física, Grupo de Pesquisa em Ciências Aplicadas ao Futebol, Campus Governador \\ Valadares, Juiz de Fora, MG, Brasil. \\ ${ }^{3}$ Associacao Esportiva e Recreativa USIPA, Departamento Gerencial das Categorias Juvenis A, \\ Ipatinga, $M G$, Brasil. \\ ${ }^{4}$ Unileste, Departamento de Educação Física, Coronel Fabriciano, MG, Brasil.
}

\begin{abstract}
Aims: The present study aimed to analyze the effects of the 3- and 6-substitution rule and qualification round on substitution pattern, game statistics, win rate, and winning probability in Brazil's largest U-20 football tournament. Methods: We analyzed 1,339 matches from the São Paulo Junior Football Cup implementing a before-after design considering 6 years (three seasons for 3 -subs and three for 6 -subs). Results: Approximately $31 \%$ of teams used the total number of replacements of 6-subs with the same substitution pattern between rounds. The win rate for both substitution rules was approximately $79 \%(\mathrm{OR}=3.10,95 \% \mathrm{CI}[2.18,4.51])$ with a small reduced magnitude (approximately 5\%) in 6-subs knockout games. Logistic regression analysis showed that the 6-subs regulation tournament predicted a reduced probability $(-12 \%)$ of a match ending with a win in the knockout round. There were 6-subs knockout effects $(p<0.05)$ on game statistics with "negligible" effect size (ES) on "effective playing time" (+1 min; ES = 0.15); decreased "goal scoring" $(-0.3 ; \mathrm{ES}=0.09)$ and "red cards" per match $(-0.1 ; \mathrm{ES}=0.06)$. An ordered probit model and partial effects showed that including a replacement unit on a team increased the winning probability by $8 \%$ in the group stage, and reduced the chance (-4\%) for the opponents trying to do the same in the knockout stage. Conclusion: Overall, 6-subs caused minor effects on the game statistics, win rate, and winning probability. Understanding substitution strategies and motivation, individual and collective performance effects, and whether there will be changes in the game profile will become relevant research issues when a greater number of substitutions rules are universally instituted.
\end{abstract}

Keywords: soccer, substitutions rule, performance analysis, coaches', decisions, strategy.

\section{Introduction}

Unlike other sports, coaches in football have limited opportunities to directly impact the game's progress. There are no timeouts and only one longer guaranteed stoppage in the game occurs at halftime in adult professional matchplay competition. For this reason and the inherent difficulty in transferring information during gameplay, coaches' interventions, for rationally exploiting and timing their allowed substitutions during the game, are critical to gain a competitive advantage when trying a more cohesive offensive/defensive strategy ${ }^{1}$ or to alleviate in-game and game-to-game fatigue, which is an important aspect during a season ${ }^{2-4}$. Thus, if the coach's decisions to replace players during matches are not correct, they will only stay in the background while the players try to adapt to the tactical, physical, and strategic flow of the game with little chance of changing the course of the match outcome.

The Fédération International of Football Association (FIFA) game rules, according to Rule 3, currently allow up to three substitutions in most competition regulations involving adult professionals during an official match and do not allow re-entry of the substituted player. However, FIFA allows up to five substitutions in international friendly matches provided both teams agree before the start of the match. Although the "three substitutions per game" rule has been consolidated for over 40 years in football, there has long been debate about changing the rules to allow more substitutions of players during the match in adult professional competitions. For exam- 
ple, the justifications in adult categories point to the preservation of the athletes' health condition by squad rotation as frequent substitutions can alleviate in-game and game-to-game fatigue ${ }^{5}$. The other reason is that limiting substitutions to three per game means fewer opportunities when football entities' policy is to encourage the participation of a larger number of people, mainly in young, student-athlete competitions ${ }^{3}$. Thus, FIFA governance and other football institutions are answering the football community's call to modify the rule regarding the substitution numbers per game, changing from 3-subs to 6-subs in tournaments of younger categories two years ago.

Substitutions are used for several reasons. For example, the first order for coaches to substitute a player is to prioritize a players' health (injured/underperforming) ${ }^{6}$ or to replace a player who has been punished with a yellow card $^{7}$, a fact which anticipates the first replacement in $\sim 9 \mathrm{~min}^{1}$. Other reasons for substitutions appear to be conditioned by contextual-related variables (e.g., match status, quality of the opposition, and match location). For example, previous studies with the 3-subs regulation have shown concordant results that changing players was based on match status (goal differences between counterparts), with teams making substitutions earlier when either tied or behind $^{1,2,4,8,9}$. Also, the quality of the opposition variable showed a positive significant effect with lower-ranked teams replacing more frequently ${ }^{3}$, and the worse the opponent the later the substitution occurs, while the better the opponent, the earlier the substitution ${ }^{1}$.

The previously mentioned substitution pattern showed that midfielders are the most substituted players ${ }^{1}$. From a tactical function standpoint, most of all substitutions (about 63\%) are "neutral" (the same playing position for a player in and player out), with similar distribution between defensive and offensive substitutions (19\% and $18 \%$, respectively) ${ }^{1}$. The coaches avoid defensive substitutions when facing a lower-ranked team - preferring defenders to enter when facing opponents of similar rank ${ }^{4}$. For match location, previous studies have reported contradictory findings, with the home team making their first substitution earlier ${ }^{12}$ or no difference in substitution timing between home and away teams ${ }^{8}$.

From the work-rate analysis point of view, important works have studied ${ }^{6,10,11}$ or reviewed ${ }^{12}$ the performance effects of substitute players on football matches. These researchers cited above demonstrated a suggestive potential for position-dependent substitutions, with an increase in physical activities performed by the substitutes to mainly cover a greater distance in high-intensity running ${ }^{6}$. Although substitutes consensually show an improvement related to physical performance, indicators of tactical activities such as pass accuracy (\%) were similar between players who entered during the match and those who started the match ${ }^{6}$. In the same away, another study showed that an early substitution improved the ball possession for the substitution team ${ }^{1}$.

Despite the lack of evidence and inconsistencies in key physical performance indicators to support coaches' decisions on player substitutions, most of them still make their substitutions during the second half to reduce the effects of fatigue across the team. Thus, the substitution pattern observed in adult professional match revealed a larger number of substitutions being made at halftime ${ }^{2}$ and mainly with a higher density on the histogram of substitutions occurring between 60 and $90 \mathrm{~min}^{12}$ in the Spanish Football League. Moreover, it is shown in the literature that changing players increases the chances of scoring more goals. For example, analyzes of 16 major world football leagues (National Championships and Cups) showed that the players who entered the match scored $\sim 11 \%$ of the goals, corresponding to $\sim 26 \%$ of the goals scored after the first substitution ${ }^{13}$. In the same study it was also noted that the second substitution had less influence on the outcome of the match, which could signal time-effectiveness issues after entering the match.

Because of the above, substitution patterns are an important debatable topic that requires more data from 3subs studies and now demands more knowledge with the implementation of 6-subs rules. Thus, considering that the six-substitution rule is not yet universally presented in professional adult football competitions, sports scientists, coaches, and fans have continued to feel apprehensive about what may be happening in younger player category tournaments that have used this new substitution rule. For example, while $50 \%$ of the starter players can be replaced in the 6-subs, the increase in the number of substitutions also offers more possibilities to influence the following game. Therefore, coaches can not only replace injured players, but also correct tactical errors, compensate for team fatigue, adapt to the evolution of the result, or retire yellow card cautioned players. Additionally, it would be generally wise to expect a technical-tactical, physiological- and performance-related effects in game statistics.

As a consequence of the new 6-subs rule implementation and hypothesized game statistics effects, the coach may be confused between conservatism and boldness. For example, on the one hand we can have important physical performance-related effects to change a team's positional platform or game strategy according to the match status. On the other hand, these last two tactical proposals can be missed by the greater number of substitutions and may cause loss of team unity. Taken together, this new profile and playing style of football matches can be sustained with decreased intra-team synchronization and coupling relations ${ }^{14,15}$ and ineffective "information transfer" theories ${ }^{16}$. Lastly, increasing the number of substitutions may put an added increase in the unpredictability of football games. Therefore, we must have more knowledge and understanding about preliminary data on 
experimental introductions of the 6-subs rule in younger player football tournaments and its effects in replacing pattern and game statistics related to effective playing time, disciplinary sanctions by referees, and the winning rate between 3 and 6-subs substitution rules and tournament stages.

While competitions with broad time-motion technology coverage and a more robust analysis of variables related to passing, organizing, and defending are not covered herein, which would allow for more in-depth and valuable studies with due discrimination by situational variables ${ }^{17}$, preliminary and simpler results may increase firsthand information. Thus, we used the most important U-20 football tournament in Brazil over 6 years to explore and improve understanding of this investigative theme, covering three seasons with 3 -subs and three seasons after the introduction of the 6-subs rule. With this approach, we firstly analyzed the effects of the two different substitution rules in tournaments and each qualification round, considering: i) substitution pattern; ii) overall winning rate and the likelihood that the match ends with a winner; and iii) game statistics (effective playing time and disciplinary sanction). Secondly, as there is an increase in the degree of freedom of substitutions numbers, we will try to estimate the effects of the number of substitutions on the probability of each match outcome.

\section{Methods}

\section{Data resource, data collection, and match sample}

The study implemented a descriptive approach and adopted official public data provided and authorized by FPF - Federação Paulista de Futebol (a member of CBF, Confederação Brasileira de Futebol). Each official match report summary of the tournament and the match statistics were taken from the public website of http://www.futebolpaulista.com.br.

To obtain a complete agreement between tabulated data and the sources, a work routine in three stages was established: First, the dataset was recorded in duplicate by two independent researchers. In the sequence, the same datapoint of the two independent files was compared in a spreadsheet to track inconsistencies in data entry (by typo, visual fatigue, etc.). After this initial screening, only $5 \%$ of the dataset showed disagreement between the researchers' data tabulation. In the third stage, the official match report summary was used again to repair the disagreements in the tabulation. This process was repeated until there was a complete agreement between the two independent researchers.

An exploratory analysis was performed after data entry agreement, which included the measures and hypothesis testing the effects of the different substitution rules on the tournament (3-subs and 6-subs). We selected the São Paulo Junior Football Cup (the largest U-20 tournament in Brazil) to conduct these analyzes because it has a highly competitive standard for young players, and it is an experimental tournament with the new 6-substitutions rule in the tournament's regulation. The regulation stipulates that a team is allowed to replace up to six (6) athletes per match, provided that it respects a maximum of three (3) substitution acts during the match, does not consider the half-time interval to count in these acts.

Throughout the tournament period (i.e., January 2 nd until January 25th), all teams are visiting teams [hosted in the same group of host cities]) in both versions of the tournament, and therefore it is possible to eliminate sources of bias such as the already known effect of the home team's winning rate, which is considered high in Brazil ${ }^{18}$. For example, other studies have shown this phenomenon from competitions with younger athletes ${ }^{19}$ to professionals in Brazilian soccer ${ }^{18,20}$. A series of match-related and favorable contextual conditions are listed when there is a home team (familiarization, attendance support, referee bias, territoriality, and psychological factors) that could interact with the substitutions and engagement of the players. Due to the traditional tournament having a high number of participating teams (the $50^{\text {th }}$ edition had 128 participants in 2019 season), we consider this tournament suitable for the descriptive purposes of this study as it is separated from the sources of bias commented above. Furthermore, the competition is the largest U-20 tournament in Brazil, involving the main clubs in Series A and B of Brazil which are the elite professional leagues, and composed of clubs from different regions in the country, including remote areas.

The sample consisted of 1,339 matches played from three previous seasons with 3 -subs $(2014,2015,2016)$ and three seasons after the 6 -subs version regulation (2017, $2018,2019)$. All teams played in the group stage in a host city. Following that first competition stage, the clubs classified in each group (the first two in classification table) stayed or moved to another host city for the subsequent knockout stage. In the eliminatory stage, the observed game results were only for the 90-min period of the match. In the U-20 São Paulo Football Cup, all matches which finished tied at this stage were decided through penalties and not by a 30-min extra-time period. The only exclusion criterion was to remove those games with dismissed players from the analysis since they were a potential source of modification of the match-related demands and dispute scenario. The study protocol was approved and followed the guidelines stated by the Ethics Committee of the local university in agreement with the ethical principles stated by the Declaration of Helsinki.

Variables and data exploration

The tabulated data frame contained: the seasons; two situational variables (tournament stages with two types of 
competition disputes [first stage, a group matches; after, a knockout stage with a round of 64, 32 and 16; quarterfinals, semi-finals, and final], and match outcome [the final result of a match]); counts on player substitutions; and game statistics (referee sanctions: red and yellow cards per team; and "effective playing time"). The "effective playing time" variable was considered as "the duration of playtime after subtracting the time taken up by stoppages (fouls, goals, throw-ins, goal kicks, injuries, etc.), meaning the amount of time during which the ball is stationary" ${ }^{21}$.

In the second step, variables derived from the initial data exploration were qualitatively coded to operationalize the hypothetical study analyzes according to a comparison of the regulation versions regarding the substitution of players in the dependent variable 'match statistics' according to the two nominal levels (3-subs or 6-subs) and tournament stages (groups/knockout). Other dependent variables were also recoded as needed, such as match outcome success in binary (win "yes" or "no") and ordered levels (win $>$ draw $>$ lost). The total number of substitutions in each team was used as a predictive variable.

Unfortunately, it was not possible to include processes involving the player and team performance (tactical, physical, technical) or other situational variables beyond the type of competition, competition round, and match outcome (i.e. quality of opposition, opposite formation). It is an amateur tournament and has logistical limitations for extensive implementation of on-site observational or even technological observational analysis for data collection. Similarly, team ability was not considered as a control variable. The tournament under consideration is multi-stage, making it difficult to establish comparative competitive ability between participants after the group stage. Moreover, there is no ranking that covers all teams or historical performances in the same tournament that would allow the team ability to be established and then do control by "quality of opposition". The opposition team formation and match status were also not taken into account, since this variable is difficult to control from the beginning of the match, considering that its complex processes can evolve during the match and that we also did not have the necessary data as argued above. Furthermore, we made no distinction as to whether a coach's substitution was for defensive, neutral, or offensive purposes.

\section{Statistical analyses}

Data are presented with frequency counts and percentages, or as mean $\pm \mathrm{SD}$ and confidence intervals at $95 \%$ level (95\% CIs) when appropriate. The Mann Whitney rank-sum test was used to compare 3- and 6-substitution rule version tournaments. Cliff's delta effect size was used for the magnitude of difference using the thresholds $|\mathrm{d}|<0.147$ as "negligible", $|\mathrm{d}|<0.33$ as "small", $|\mathrm{d}|<0.474$ as "medium", and over as "large" 22 . The Spearman test (rho) was used to verify the correlation between the variables. A binary logistic regression analysis was applied to ascertain the effects of substitutionregulation and tournament dispute stages on the likelihood that the match ends with a winner. We regressed the match outcomes (win $>$ draw $>$ lost) on the team's probability of winning according to the number of substitutions made by each competing team and term interaction using an ordered probit model with proportional odds. The odds ratio (OR) with corresponding 95\% CIs were reported. All statistical analyses were performed using R (R Core Team, version 3.6.0). The statistical significance was set at $p<0.05$ for all analyses.

\section{Results}

\section{Descriptive results of quantitative replacing and match outcomes rates in two substitution-regulation versions of the tournament}

The quantitative replacing (count and proportion of teams that used a certain number of substitutions) is

Table 1 - Quantitative substitution per team in terms of two substitution-regulation of the tournament as per the tournament dispute stage.

\begin{tabular}{|c|c|c|c|c|c|c|}
\hline \multirow[b]{3}{*}{ Quantitative subs } & \multicolumn{6}{|c|}{ Substitution-regulation tournaments } \\
\hline & \multicolumn{3}{|c|}{ 3-subs } & \multicolumn{3}{|c|}{ 6-subs } \\
\hline & $\begin{array}{c}\text { Group stage } \\
(\mathrm{n}=160) /\end{array}$ & $\begin{array}{c}\text { Knockout stage } \\
(\mathrm{n}=438)\end{array}$ & Overall $(n=598)$ & $\begin{array}{c}\text { Group stage } \\
(\mathrm{n}=188)\end{array}$ & $\begin{array}{c}\text { Knockout stage } \\
((n=553)\end{array}$ & $\begin{array}{l}\text { Overall } \\
(\mathrm{n}=741)\end{array}$ \\
\hline 0 & $0(0 \%)$ & $0(0 \%)$ & $0(0 \%)$ & $1(0.3 \%)$ & $2(0.2 \%)$ & $3(0.2 \%)$ \\
\hline 1 & $0(0 \%)$ & $9(1 \%)$ & $9(0.8 \%)$ & $0(0 \%)$ & $4(0.4 \%)$ & $4(0.3 \%)$ \\
\hline 2 & $14(4.4 \%)$ & $58(6.6 \%)$ & $72(6.0 \%)$ & $6(1.3 \%)$ & $35(3.2 \%)$ & $41(2.8 \%)$ \\
\hline 3 & $306(95.6 \%)$ & $809(92.4 \%)$ & $1115(93.2 \%)$ & $48(12.8 \%)$ & $158(14.3 \%)$ & $206(13.9 \%)$ \\
\hline 4 & & & & $94(25.0 \%)$ & $280(25.3 \%)$ & $374(25.2 \%)$ \\
\hline 5 & & & & $107(28.5 \%)$ & $288(26.0 \%)$ & $395(26.7 \%)$ \\
\hline 6 & & & & $120(31.9 \%)$ & $339(30.7 \%)$ & $459(31.0 \%)$ \\
\hline
\end{tabular}

Data are counts and relative proportions (\%). Subs: substitutes. 3-subs and 6-subs: maximum limit of three and six players in the two versions of the substitution regulations of the tournament, respectively. Group and Knockout represent the tournament dispute stages. 
Table 2 - Quantitative replacing (frequency and relative proportion) per matches in terms of two substitution-regulation per stage of tournament dispute stages.

\begin{tabular}{|c|c|c|c|c|c|c|}
\hline \multirow[b]{3}{*}{ Quantitative subs } & \multicolumn{6}{|c|}{ Substitution-regulation tournaments } \\
\hline & \multicolumn{3}{|c|}{ 3-subs } & \multicolumn{3}{|c|}{ 6-subs } \\
\hline & $\begin{array}{c}\text { Group stage } \\
(\mathrm{n}=160)\end{array}$ & $\begin{array}{c}\text { Knockout stage } \\
(\mathrm{n}=438)\end{array}$ & $\begin{array}{c}\text { Overall } \\
(\mathrm{n}=598)\end{array}$ & $\begin{array}{c}\text { Group stage } \\
(\mathrm{n}=188)\end{array}$ & $\begin{array}{c}\text { Knockout stage } \\
\quad(n=553)\end{array}$ & $\begin{array}{c}\text { Overall } \\
(\mathrm{n}=741)\end{array}$ \\
\hline 0 & $0(0 \%)$ & $0(0 \%)$ & $0(0 \%)$ & $0(\%)$ & $0(\%)$ & $0(\%)$ \\
\hline 1 & $0(0 \%)$ & $0(0 \%)$ & $0(0 \%)$ & $0(\%)$ & $0(\%)$ & $0(\%)$ \\
\hline 2 & $0(0 \%)$ & $0(0 \%)$ & $0(0 \%)$ & $0(\%)$ & $0(\%)$ & $0(\%)$ \\
\hline 3 & $0(0 \%)$ & $1(0.2 \%)$ & $1(0.2 \%)$ & $0(\%)$ & $0(\%)$ & $0(\%)$ \\
\hline 4 & $1(0.6 \%)$ & $11(2.5 \%)$ & $12(2.0 \%)$ & $0(\%)$ & $0(\%)$ & $0(\%)$ \\
\hline 5 & $12(7.5 \%)$ & $51(11.6 \%)$ & $63(10.5 \%)$ & $0(0 \%)$ & $7(1.3 \%)$ & $7(0.9 \%)$ \\
\hline 6 & $147(91.9 \%)$ & $375(85.6 \%)$ & $522(87.3 \%)$ & $9(4.8 \%)$ & $27(4.9 \%)$ & $36(4.9 \%)$ \\
\hline 7 & & & & $16(8.5 \%)$ & $56(10.1 \%)$ & 72 (9.7\%) \\
\hline 8 & & & & $27(14.4 \%)$ & $94(17.0 \%)$ & $121(16.3 \%)$ \\
\hline 9 & & & & $39(20.7 \%)$ & $117(21.2 \%)$ & $156(21.1 \%)$ \\
\hline 10 & & & & $38(20.2 \%)$ & $90(16.3 \%)$ & $128(17.3 \%)$ \\
\hline 11 & & & & $34(18.1 \%)$ & $96(17.4 \%)$ & $130(17.5 \%)$ \\
\hline 12 & & & & $25(13.3 \%)$ & $66(11.9 \%)$ & $91(12.3 \%)$ \\
\hline
\end{tabular}

Data are frequency and relative proportion (\%). Subs: substitutes. 3-subs and 6-subs: maximum limit of three- and six-players changes in the two substitution regulations of the tournament, respectively. Group and Knockout represent the tournament dispute stages.

shown in Table 1. Table 2 shows the frequency and proportion of games with the particular number of substitutions of each substitution regulation and tournament dispute stages. About $93 \%$ of the teams in the 3 -subs condition used the maximum of the 3 allowed substitutions, corresponding to $\sim 98 \%$ of the matches with a total of 5 and 6-substitutions (Table 1). On the other hand, in the 6subs version it was observed that the total use of substitutions occurred in only $\sim 31 \%$ of the teams, and only $\sim 12 \%$ of matches had a permitted total of 12 players replaced (Table 2). The quantitative substitution of players followed the same counting pattern between the group and knockout tournament dispute stages in both substitutionversion tournaments.

A binary logistic regression was performed to ascertain the effects of the substitution-regulation and tournament round on the likelihood that the match ends with a winner (Table 3). Only the model including the substitution-version (6-subs as the referenced level) and tournament dispute stages (group or knockout) as mains effects with the interaction term "subs regulation: stage of dispute" was significant $\left(\chi_{1}^{2}=5.29, p<0.05\right)$. The partial effects or discrete change analysis showed that a game in a 6-subs regulation tournament in the knockout phase was associated with a reduced probability $(\sim-12 \%)$ of a match ending with a winning result. In short, the simple contrast revealed diagonally opposite cell rates, leading to a win rate difference between the subs versions stratified for stages (about $\sim+6.1 \%$ [win counts $=121 / 160$ games $=$ $75.6 \%$ to $358 / 438$ games $=81.7 \%]$ and $\sim-5.5 \%$ win counts $=155 / 188$ games $=82.4 \%$ to $425 / 553$ games $=76.9 \%]$ ) for 3 -subs and 6-subs, respectively. The win rate in the two subs regulation was $\sim 79 \%(\mathrm{OR}=3.10,95 \%$ CI $[2.18$, 4.51]).

\section{The overall effect of two substitution-regulation versions of the tournament on match statistics}

Table 4 shows the descriptive results derived from subs-version comparisons on match statistics variables. The 6-subs in the knockout stage had approximately one

Table 3 - Parameter estimates fitting a logistic regression model with a binary match outcome (win or draw) as the dependent variable.

\begin{tabular}{lcc}
\hline Exploratory variables included & $\boldsymbol{\beta}(\mathbf{S E})$ & Partial effects $\boldsymbol{d} \boldsymbol{F} / \boldsymbol{d} \boldsymbol{x}(\boldsymbol{S} \boldsymbol{E})[\mathbf{z}$ value, $\boldsymbol{p}>|\mathbf{z}|]$ \\
\hline Subs regularion (6-subs) & $0.415(0.266)$ & $0.069(0.05)[1.54,0.123]$ \\
Stage (knockout) & $0.366(0.222)$ & $0.063(0.04)[1.58,0.114]$ \\
Subs regulation (6-subs):Stage(knockout) & $-0.713(0.310)^{*}$ & $-0.122(0.05)[-2.24,0.025]^{*}$ \\
\hline
\end{tabular}

Subs version: substitution conditions (3-subs and 6-subs, maximum limit of three and six players in the substitution regulation of the tournaments, respectively). Stage represents "groups" and "knockout" of tournament dispute stages. Dependent variable: match outcome, the win level "Yes" coded as reference response (class 1). $\beta(\mathrm{SE})$ : estimated coefficients and (standard errors). Constant $=1.132(0.184), p<0.001 . \mathrm{R}^{2}=0.004(\mathrm{McFadden})$. Sub regulation:Stage $=$ significant interaction term included in model $\left(\chi_{1}^{2}=5.29, p=0.02\right) .{ }^{*} p<0.05$. 
Table 4 - Comparison of match statistics in terms of two substitution-regulation of the tournament round.

\begin{tabular}{|c|c|c|c|c|c|c|}
\hline \multirow[t]{2}{*}{ Match statistics } & \multirow[t]{2}{*}{ Stage } & \multicolumn{2}{|c|}{ Tournaments } & \multirow[t]{2}{*}{$\chi_{1}^{2}$} & \multirow[t]{2}{*}{$p$-value } & \multirow[t]{2}{*}{$\operatorname{ES}[95 \% \mathrm{CI}]$} \\
\hline & & 3-subs & 6-subs & & & \\
\hline \multirow[t]{2}{*}{ Effective playing time ( $\mathrm{min} /$ per match) } & Group & $61 \pm 3$ & $62 \pm 3$ & 2.364 & 0.124 & $0.09[-0.02,0.21]$ \\
\hline & Knockout & $61 \pm 3$ & $62 \pm 3$ & 17.137 & 0.001 & $0.15[0.08,0.22]$ \\
\hline \multirow[t]{2}{*}{ Score (per match) } & Group & $3.1 \pm 2$ & $2.9 \pm 2$ & 1.693 & 0.193 & $0.08[-0.04,0.20]$ \\
\hline & Knockout & $3.2 \pm 2$ & $2.9 \pm 2$ & 6.301 & 0.012 & $0.09[0.02,0.16]$ \\
\hline \multirow[t]{2}{*}{ Fouls committed (per match) } & Group & $26 \pm 6$ & $25 \pm 6$ & 1.373 & 0.241 & $0.07[-0.05,0.19]$ \\
\hline & Knockout & $26 \pm 6$ & $26 \pm 6$ & 0.124 & 0.723 & $0.01[-0.06,0.08]$ \\
\hline \multirow[t]{2}{*}{ Yellow cards (per match) } & Group & $3.9 \pm 2$ & $3.6 \pm 2$ & 0.404 & 0.524 & $0.04[-0.08,0.15]$ \\
\hline & Knockout & $4.1 \pm 2$ & $4.0 \pm 2$ & 1.396 & 0.237 & $0.04[-0.03,0.11]$ \\
\hline \multirow[t]{2}{*}{ Red cards (per match) } & Group & $0.3 \pm 0.6$ & $0.2 \pm 0.5$ & 0.112 & 0.737 & $0.14[-0.06,0.09]$ \\
\hline & Knockout & $0.4 \pm 0.6$ & $0.3 \pm 0.6$ & 5.099 & 0.023 & $0.06[0.01,0.11]$ \\
\hline
\end{tabular}

Data are mean \pm SD. The stage represents "groups" and "knockout" of tournament dispute stages. 3-subs and 6-subs: maximum limit of three and six players in the tournament's substitution regulation, respectively. $\chi_{1}^{2}$ : chi-squared test with $\mathrm{df}=1$. The Mann Whitney test was used to compare the twosubstitution regulation. Effects size (ES) estimate of Cliff's delta. 95\% CI (confidence intervals).

more minute of "effective playing time" with "small" effect size $\left(\chi_{1}^{2}=17.137, p<0.001, \mathrm{ES}=0.15\right)$; a decrease in average "score per game" $\left(\chi_{1}^{2}=6.301, p<0.05, \mathrm{ES}=\right.$ $0.09)$ and one-tenth in "red cards" $\left(\chi_{1}^{2}=5.009, p<0.05\right.$, $\mathrm{ES}=0.06$ ), with the last two differences having a "negligible" effect size.

\section{Relationship between the number of substitutions and match statistics indicators, and the effects of substitution numbers on the outcome}

We evaluated the relationship between the number of subs and match statistics indicators to check the robustness of our findings (Table 4). We also analyzed the impacts of the number of substitutions made on the probability of ordered match outcome (winning, drawing, and losing results). We excluded all the matches in which the players were dismissed to aim for more control in theses aforementioned analyses (stratified sample comprised 1,046 games; equivalent to $78 \%$ of all games played).

From this stratified data, the Spearman's rank correlation (rho) revealed to have a significant correlation between the "total number of substitutions" in the match and the following indicators: "effective playing time" $(\mathrm{min} /$ per match; rho $=0.11, p<0.001,95 \%$ CI $[0.05$, $0.17]$; and "total score" (rho $=0.06,95 \%$ CI [0.01, 0.12], $p<0.03$ ). The indicators "yellow card per match" (rho = $-0.01,95 \%$ CI $[-0.08,0.04])$ and "fouls per match" (rho = $-0.05,95 \%$ CI $[-0.10,0.01])$ were negatively correlated, but without statistical significance ( $p=0.52$ and $p=0.13$, respectively). A significant positive correlation was only observed between the "total numbers of substitutions" and "total score per match" (rho $=0.28,95 \%$ CI $[0.20,0.35]$, $p<0.001)$ when the data was again sub-stratified by matches with more than six substitutions $(\mathrm{N}=560)$. In the same sub-stratification, the indicators "effective playing time", "foul committed", and "yellow card" were not sig- nificantly correlated with "total substitutions in the game" (rho $=0.06,95 \%$ CI $[-0.02,0.14], p=0.17$; rho $=-0.05$, $95 \%$ CI $[-0.13,0.03], p=0.23$; rho $=-0.04,95 \%$ CI $[-0.12$, $0.04], p=0.30$; respectively).

Table 5 shows the estimated model for the outcome with ordered probit, considering the same aforementioned data matrix excluding all matches with red cards and subset per stage for this analysis. The exploratory model in the group stage showed the main effects of the number of substitutions on their team (Model 1, $\chi^{2}{ }_{1}=7.59, p<0.01$ ). The partial effects showed that when the team itself increments a unit in substitution in the group stage, there is an $8 \%$ increase in the probability of winning $(\mathrm{OR}=1.26$, $95 \%$ CI $[1.06,1.42])$ with all other variables in the model remaining constant. The number of subs in the knockout stage were the main effects (Model 2: $\chi^{2}{ }_{1}=5.70, p<0.05$; $\chi_{1}^{2}=8.07, p<0.01$, on the same and opposite team, respectively), showing the opposite scenario in the probability of winning. Then, increments in a unit in substitution on their team generate a $3 \%$ increase in the probability of winning $(\mathrm{OR}=1.09,95 \% \mathrm{CI}[1.02,1.19])$. In contrast, a unit in substitution on the opposing team decreases their probability of winning $(\mathrm{OR}=0.89,95 \% \mathrm{CI}$ $[0.83,0.97])$.

A subset by substitution differences between teams (i.e. delta) revealed a decrease in the win rate, regardless of the tournament dispute stages, when the favorable substitution delta increases (i.e. equal, delta sub+1, +2 and $+3 ; \sim 65 \%, \sim 20 \%, \sim 10 \%$ and, $\sim 5 \%$, respectively).

\section{Discussion}

The 6-substitution rule (still only allowed in youth tournaments) increases the number of substitutions, offering a wide range of strategic possibilities for coaching intervention. As a result, it is wise to think of a new game 
Table 5 - Parameter estimates fitting an ordered probit model with match outcome (win $>$ draw $>$ lost) as the dependent variable in each tournament round.

\begin{tabular}{llcc}
\hline Exploratory variables included & $\boldsymbol{\beta}(\mathbf{S E})$ & Partial effects for the outcome WIN $\boldsymbol{d F} / \boldsymbol{d} \boldsymbol{x}(\mathbf{S E})[\mathbf{z}$ value, $\boldsymbol{p}>|\mathbf{z}|]$ \\
\hline Model 1: & Group stage $(\mathrm{N}=284)$ & & \\
& Number of subs (same team) & $0.203(0.07) * *$ & $0.08(0.03)[2.76,0.006]$ \\
& Number of subs (opposite team) & $-0.070(0.07)$ & $-0.03(0.03)[-0.92,0.356]$ \\
& Intercepts (Thresholds) & & {$[-1.449,0.147]$} \\
& Lost/Draw & $-0.399(0.27)$ & {$[0.91,0.358]$} \\
& Draw/Win & $0.252(0.27)$ & $0.03(0.01)[2.43,0.015]$ \\
Model 2: & Knockout stage (N = 762) & $0.094(0.038) *$ & $-0.04(0.01)[-2.83,0.004]$ \\
& Number of subs (same team) & $-0.108(0.038) * *$ & {$[-1.57,0.116]$} \\
& Number of subs (opposite team) & & {$[1.91,0.056]$} \\
\hline
\end{tabular}

Subs: substitution numbers. The stage represents "groups" and "knockout" of tournament dispute stages. Dependent variable: match outcome, the level was coded as ordered (win $>$ draw $>$ lost). $\beta(\mathrm{SE})$ : estimated coefficients and (standard errors). McFadden $\mathrm{R}^{2}=0.02$ and 0.01 , respectively for model 1 and 2 . AIC $=533$ and 1616, respectively for Model 1 and $2 . * *, *$ indicate $p<0.01$ and $p<0.05$, respectively.

profile concerning traditional 3 -subs, differently influencing the various aspects related to individual and collective performance which would culminate in greater unpredictability in the game. In accordance with this premise, our work aims to analyze the effects of the 3-subs and 6-subs regulations (U-20 football tournament) and each round of dispute on substitution pattern, game statistics, win rate, and winning probability.

Among the main results, we noted that coaches did not use all the permitted substitutions and that the substitution pattern behavior was similar between the two types of tournaments (substitution regulation) and dispute stages. Another important reported result is that there is a decrease in the winning rate $(\sim-6 \%)$ with reduced probability $(\sim-12 \%)$ of a match ending with a win in the knockout stage in the 6-subs format. Our model estimation also pointed out that including a substitution unit in the team itself increased the chance of winning by $8 \%$ in the group stage, with the opposite happening for opponents in the knockout stage when they include one unit of player substitution ( $\sim-4 \%$ of chance of winning the match). We specifically observed a 6-subs effect in the knockout stage for game statistics with increased "effective playing time", deceased red cards, and score per match.

In paralleling the elite leagues, we noted that this youth tournament adopts a similar number of substitutions when considering the three seasons analyzed for the 3subs version of the tournament ( $\sim 87 \%$, overall stages) compared to the majority of teams across the top professional competitions $(\sim 82 \%)$ which use all three substitutions permitted ${ }^{8}$. However, only $30 \%$ of teams used all substitutions permitted in the 6-subs version, and only $12 \%$ of games had the maximum possible total of 12 substitutions take place.
Several reasons may be making it difficult to implement all permitted substitutions. The regulation of substitutions themselves occurring in no more than three (3) acts during the match can put the coach in a context of extreme doubt. For example, the coach may wish for a fifth substitution but does not do so for fear of having to make the sixth substitution together. Thus, he may be left without an option for other moments of interest in the game, or when a player's clinical substitution needs to occur. Other coaches may believe that interfering with the rhythm of the game can also be disruptive and that their team can fit in the substitution at any time. There may also be a sense from coaches and athletes that substitutes may experience negative emotions ${ }^{12}$. Therefore, pre-game preparatory strategies must be effectively designed to not only meet physical demands but also emotional ones for the game. In addition, only three acts for substitutions were permitted which may be an impediment to further fully use the substitution number. Thus, if the desire is to increase squad rotation or player participation, alternative regulations need to be studied and tested with those used in the NCAA which get good participation results with a short competitive calendar ${ }^{3}$.

Although it is difficult to make a comparison with other sports because many of them have unlimited and any-time substitution, it is noteworthy that basketball substitutions sustain a high work rate and have been considered effective in team performance improvement ${ }^{23}$. Unlike indoor team sports (handball, futsal, basketball), football coaches are left to cover a large playing field for instruction while they can only quickly attend the technical area. Thus, an inefficiency in information transfer and communication occurs in football between the coach and players. In this sense, making a large number of substitu- 
tions in a football game may point to the insecurity of modifying the game plan. Psychological reasons are associated with non-rational thinking of coaches ${ }^{24}$ and may also explain the non-use of all permitted substitutions. Thus, this would include well-known behavioral biases such as the heuristic of not changing a winning team ${ }^{25}$, or by adopting neutral substitutions in light of a new rule as noted in the introduction of the third substitution in the 1995-96 Italian Serie A season ${ }^{24}$.

Other theoretical reasons are also pointed out for coaching conservatism in substitutions. For example, removing athletes from their habituation, hindering the effectiveness of "information transfer", , and establishing networks can diminish the self-organization dynamic and overall performance of the team ${ }^{15}$. Perhaps because of these theories, and still depending on match status, some researchers have not evidenced the advantages of substitutions in key offensive performance metrics such as goal kicks and ball possession ${ }^{1}$. As a consequence, these arguments together predispose the coach to still feel uncomfortable about performing too many substitutions as the competition progresses and teams with strong ability are emerging. Therefore, they only attempt a new substitution if the match status is disadvantageous, and if this attempt is extremely necessary at the knockout stage where the team is eliminated.

The present study demonstrates a decreased likelihood that the match ends with a winner $(\sim 12 \%)$ in the knockout stage in the 6-subs. Furthermore, our estimate through the ordered probit model pointed to a reduced probability of winning as a new substitute is included. Taken together, these findings in the first order demonstrate that coaches seem to replace in reaction to unfavorable match status. On the other hand, speculation that loss of "team unity" may be occurring with each new substitution gains strength, being anchored in the aforementioned "team-mate relations" and "information transfer" theories $^{14-16}$. The caveat for these estimates seems to be the group stage observation, where coaches can rotate their squad of players when a favorable outcome seems more certain (i.e. they are leading the match by 2 or more goals). Future research may be devoted to incorporating more factors that affect substitutions beyond trying to create a substitution cut-off point that can lead to better match outcome results than reported in the present paper.

Although no physical performance-related metrics could be included in the present work to more appropriately discuss the game statistics, the findings show $\sim 1$ min more in "effective playing time" in 6-subs. On the other hand, a decrease in red cards may also be expected (as noted) because this larger number of substitutions can be used to remove those players with a yellow card before they commit another cardable foul.

Another point to consider is that a decrease in average goals $(\sim-10 \%)$ seems to be a natural consequence due to less physical exhaustion. The curious thing is that these previously mentioned game statistics were only different for the 6-subs in the knockout stage. The coaches riskless in their interventions in this stage, and it could be more accurate when introducing a substitute player. Likewise, this substitute could be better understanding their importance and as a consequence have greater physical engagement. After all, the differences noted in game-related statistics had a small magnitude between 3- and 6-subs. Furthermore, most of the match profiling-related statistical variables studied do not appear to be correlated with the final number of substitutions in the game. Only the total goals per match had ordinal growth as the total number of substitutions was increased in the game. Perhaps this once again demonstrates the reactive intervention of the coach and that exchanges could be numerous if the match status presents a great score-line differential.

Despite the important findings, the present study has a limitation of the likely potential influence of the 3-and 6-subs game-response. For example, other contextual variables were uncontrolled herein such as quality of opposition, and opponent tactics may be included in further studies because "situational variables" are crucial for gaining better knowledge ${ }^{17}$. Several other performance indicators cannot be considered because match-analytics companies did not cover this tournament and the implementation of in-situ match analysis by observation and a GPS-tracking system was not possible due to a large number of games happening simultaneously. Furthermore, this paper did not analyze the substitution time, playing position, and changes if two or more players substituted simultaneously, or the performance of each substitute player and the team as a whole. We understand that there are numerous confounding factors if there is any connection between the player introduction and team performance improvement and match status or outcome modification. These analyzes are complex and have become hard-to-reach. Multivariate modeling was not possible due to the already mentioned limitation of control variables.

Future studies may consider GPS device-based metrics to compare the two substitution rules because it is easier to implement. With this in mind, it will be easier to determine the overall impact of each substitute when introduced and the potential changes that 6-subs may cause in match demands in different athlete levels. Although 6-subs is a new fact in the literature, the effects of 3-subs and various aspects are still controversial or unknown and may be important fields of investigation as alternative match-day strategies and specific competitive balance effects in different tournament types. In addition, hot topics could be re-debated int the new 6-subs scenario as players operationalize the "conscious or subconscious pacing strategy" 26 ; because substitute players cannot exceed the running performance that they typically adopt 
during the first half of matches in which they are starting players ${ }^{6,10}$; whether soccer substitutes are physically and mentally "ready" to perform immediately upon entering the field ${ }^{12}$, or if actual pitch-entry and re-warm up are appropriate for developing the coping strategies and allow substitutes to maintain task-focus ${ }^{27}$. Thus, the referenced injury rate, technical-tactical metrics, between-match variability in reported tasks demands ${ }^{28,29}$, and proportion of goals scored during the final $15 \mathrm{~min}$ of soccer matches ${ }^{26}$ will be subject to a new magnitude and tempo-transient changes in game demands or home advantage changes ${ }^{16,19,20}$ with 6 -subs regulation and these factors should be studied.

\section{Conclusion}

Our data provide preliminary information about different player substitution number rules using the 3- (previous season) and 6-substitution (recently introduced) regulations in the same U-20 football tournament. It was observed that the win rate and some game-related statistics were slightly changed in the 6-subs, notably in the knockout stage. An increase in "effective playing time" occurred in the 6-sub version, as well as fewer "red cards", but lower "goals average per match". From match probability, it appears that the chance of winning increases with each player replacement unit in the team itself in the group stage. In the knockout stage, there is a decreased winning chance for each player unit exchange on the opposite team. Quantitative replacing analyzes showed that coaches still seem conservative and do not use all the permitted substitutions. These preliminary findings may draw attention to this new context of football match profiling. While this information may be important, more work still needs to be done on the overall impact of 6-substitutions from a technical and physical standpoint, coach's motivations and strategies for changing players, and game unpredictability. New approaches to coaching, preparation, and pre-pitch entry should be considered with other match-related profiling if a new subs regulation emerges in football.

\section{References}

1. Gomez M, Lago-Peñas C, Owen LA. The influence of substitutions on elite soccer teams' performance. Int J Sports Physiol Perform. 2016;16: 553-568.

2. Del Corral J, Barros CP, Prieto-Rodriguez J. The determinants of soccer player substitutions: a survival analysis of the Spanish soccer league. J Sports Econom. 2008;9: 160172.

3. Conte D, Niederhausen M, LaPlante M, and Tessitore A. Substitution patterns and analysis in men's Division I college soccer. In: International Research in Science and Soccer II: Routledge, 2015. pp. 44-52.

4. Rey E, Lago-Ballesteros J, Padrón-Cabo A. Timing and tactical analysis of player substitutions in the UEFA Cham- pions League. Int J Sports Physiol Perform. 2015;15: 840850.

5. Carling C, Le Gall F, Dupont G. Are physical performance and injury risk in a professional soccer team in match-play affected over a prolonged period of fixture congestion? Int $\mathrm{J}$ Sports Med. 2012;33: 36-42.

6. Bradley PS, Lago-Peñas C, Rey E. Evaluation of the match performances of substitution players in elite soccer. Int $\mathrm{J}$ Sports Physiol Perform. 2014;9: 415-424.

7. Ascari G, Gagnepain P. Spanish football. Journal of sports economics. 2006;7: 76-89.

8. Myers BR. A proposed decision rule for the timing of soccer substitutions. J Quant Anal Sports. 2012;8: 1-24.

9. Hirotsu N, Ito M, Miyaji C, Hamano K, Taguchi A. Modeling tactical changes of formation in association football as a non-zero-sum game. J Quant Anal Sports. 2009;5.

10. Carling C, Espié V, Le Gall F, Bloomfield J, Jullien H. Work-rate of substitutes in elite soccer: A preliminary study. J Sci Med Sport. 2010;13: 253-255.

11. Hills SP, Barrett S, Feltbower RG, Barwood MJ, Radcliffe $\mathrm{JN}$, Cooke CB, et al. A match-day analysis of the movement profiles of substitutes from a professional soccer club before and after pitch-entry. PLoS One. 2019;14: e0211563.

12. Hills SP, Barwood MJ, Radcliffe JN, Cooke CB, Kilduff LP, Cook CJ, et al. Profiling the responses of soccer substitutes: A review of current literature. Sports Med. 2018;48: 22552269.

13. Flôres FS, dos Santos DL, Carlson GR, Gelain EZ. What can coaches do? The relationship between substitution and results of professional soccer matches. RBFF-Revista Brasileira de Futsal e Futebol. 2019;11: 215-222.

14. Goncalves BV, Figueira BE, Macas V, Sampaio J. Effect of player position on movement behaviour, physical and physiological performances during an 11-a-side football game. J Sports Sci. 2014;32: 191-199.

15. Folgado H, Duarte R, Fernandes O, Sampaio J. Competing with lower-level opponents decreases intra-team movement synchronization and time-motion demands during pre-season soccer matches. PLoS One. 2014;9: e97145.

16. Tsonis AA, Tsonis PA. Information Transfer and Home Field Advantage. Mathematics Today. 2001;37: 24-25.

17. Gómez M, Lago-Peñas C, and Pollard R. Situational variables. In: Routledge handbook of sports performance analysis. London and New York: Routledge, 2013. pp. 277-287.

18. Pollard R, Silva CD, Medeiros NC. Home advantage in football in Brazil: differences between teams and the effects of distance traveled. Braz J Soccer Sci. 2008;1: 03-10.

19. Pollard R, Gómez MA. Comparison of home advantage in college and professional team sports in the United States. Coll Antropol. 2015;39: 583-589.

20. Drummond LR, Drummond FR, Silva CD. The home advantage in soccer: comparison between Libertadores of American Cup and UEFA Champions League. Rev Bras Educ Fís Esporte. 2014;28: 283-292.

21. Casamichana D, Castellano J. Time-motion, heart rate, perceptual, and motor behaviour demands in small-sides soccer games: Effects of pitch size. J Sports Sci. 2010;28: 16151623.

22. Romano J, Kromrey JD, Coraggio J, Skowronek J. Appropriate statistics for ordinal level data: Should we really be 
using t-test and Cohen'sd for evaluating group differences on the NSSE and other surveys. S1-33, 2006.

23. Gomez M, Silva R, Lorenzo A, Kreivyte R, Sampaio J. Exploring the effects of substituting basketball players in high-level teams. J Sports Sci. 2017;35: 247-254.

24. Varela-Quintana C, del Corral Cuervo J, Prieto-Rodríguez J. The effect of an additional substitution in association football. Evidence from the Italian Serie A. Rev Psicol Deporte. 2016;25: 101-105.

25. Nüesch S, Haas H. Empirical evidence on the "Never Change a Winning Team" heuristic. Jahrbücher für Nationalökonomie und Statistik. 2012;232: 247-257.

26. Reilly T. Energetics of high-intensity exercise (soccer) with particular reference to fatigue. J Sports Sci. 1997;15: 257263.

27. Woods B, Thatcher J. A qualitative exploration of substitutes' experiences in soccer. Sport Psychol. 2009;23: 451469.

28. Gregson W, Drust B, Atkinson G, Salvo VD. Match-tomatch variability of high-speed activities in premier league soccer. Int J Sports Med. 2010;31: 237-242.
29. Russell M, Northeast J, Atkinson G, Shearer DA, Sparkes $\mathrm{W}$, Cook CJ, et al. Between-match variability of peak power output and creatine kinase responses to soccer match-play. J Strength Cond Res. 2015;29: 2079-2085.

\section{Corresponding author}

Carlos Fernando Braz Ribeiro. Universidade Federal de Viçosa, Centro de Ciências Biológicas, Departamento de Educação Física, Curso de Especialização de Futebol, Viçosa, MG, Brasil.

E-mail: carlosbraz22@hotmail.com

Manuscript received on October 25, 2019

Manuscript accepted on April 3, 2020

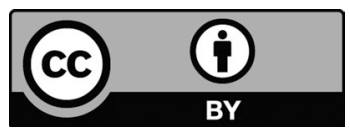

Motriz. The Journal of Physical Education. UNESP. Rio Claro, SP, Brazil - eISSN: 1980-6574 - under a license Creative Commons - Version 4.0 